\title{
Nonlinear Schrödinger equation with power-law confining potential
}

\author{
Sheila M. Holz, ${ }^{1}$ Victo S. Filho, ${ }^{1}$ Lauro Tomio, ${ }^{1}$ and A. Gammal ${ }^{2}$ \\ ${ }^{1}$ Instituto de Física Teórica, Universidade Estadual Paulista, 01405-900, São Paulo, Brazil \\ ${ }^{2}$ Instituto de Física, Universidade de São Paulo, 05315-970, C.P. 66318, São Paulo, Brazil
}

(Received 21 March 2006; published 19 July 2006)

\begin{abstract}
Critical limits of a stationary nonlinear three-dimensional Schrödinger equation with confining power-law potentials $\left(\sim r^{\alpha}\right)$ are obtained using spherical symmetry. When the nonlinearity is given by an attractive two-body interaction (negative cubic term), it is shown how the maximum number of particles $N_{c}$ in the trap increases as $\alpha$ decreases. With a negative cubic and positive quintic terms we study a first order phase transition, that occurs if the strength $g_{3}$ of the quintic term is less than a critical value $g_{3 c}$. At the phase transition, the behavior of $g_{3 c}$ with respect to $\alpha$ is given by $g_{3 c} \sim 0.0036+0.0251 / \alpha+0.0088 / \alpha^{2}$.
\end{abstract}

DOI: 10.1103/PhysRevA.74.013406

\section{INTRODUCTION}

Nonlinear Schrödinger equations (NLSE) have been considered in applications to several branches of physics, as nonlinear waves, fluid dynamics, optics, superconductivity, superfluidity, and Bose condensation (see, for example, some reviews in Refs. [1-4]). One of the most important variations of NLSE, in which the nonlinear term is cubic, was developed from works of Ginszburg, Pitaevskii, and Gross [5], actually known as Gross-Pitaevskii (GP) equation. This equation was discussed by different authors in the years 90 in the context of BEC [6], turbulence in fluids and superfluids [7]. It has been used to describe atomic condensation in the weakly interacting regime, in the mean-field approximation, providing numerical results close to experimental data.

The experimental realization of Bose-Einstein condensation (BEC), for trapped atomic systems with positive [8] and negative [9] two-body scattering length $a$, brought enormous interest in this subject. Such results have motivated plenty of works treating the GP equation with harmonic traps in spherical or cylindrical symmetry (see, for instance, Refs. $[2,4,10-13])$. The case of atomic systems with two-body attractive interactions is of particular interest, as the condensate becomes unstable when the number of condensed particles overcomes a critical number $N_{c}$. So, in several recent works the authors are concerned with measurements and calculations of this critical value using different geometries for the trap (see Ref. [14] and references therein). Actually, relevant phenomena for systems with $a<0$ are been experimentally investigated and theoretically analyzed, as for example the collapse-revival processes [15-19]. One of the interesting aspects of such systems is concerned with the possibility of increasing the critical number $N_{c}$ of condensed atoms, trapped by harmonic interactions. This can be realized by changing the symmetry of the trap, through different components of the frequencies of the three-dimensional (3D) harmonic potential, as discussed in Refs. [20,21]. Another way to increase $N_{c}$ can be done by modifying the shape of the trapping potential [22].

Studies with power-law trapping potentials were realized by several authors [23-29]. In Ref. [23], the authors calculated the critical temperature $T_{c}$ of Bose-Einstein condensates trapped by such a potential and analyzed in detail its thermodynamics. A recent analysis is also done in Ref. [29]. The possibility of obtaining a phase transition to a condensed
PACS number(s): 32.80.Pj, 03.75.-b, 36.40.-c, 34.10.+x

state, for alkali dilute gases, was studied in Ref. [24] by considering a confining power-law potential, which is changed adiabatically. References [25-28] analyze the behavior of condensates trapped by power-law potentials in arbitrary dimensions. In a one-dimensional Bose gas subjected to a potential of the form $V=V_{0}(|x| / a)^{\gamma}$, a significant number of particles in the lowest energy state was found for $\gamma<2$ in Ref. [26]. Further studies about condensates trapped by power-law potentials can be found in Refs. [30-33]. However, none of these studies with nonharmonic power-law trapping potentials have considered the limitations in the maximum number of condensed atoms, in case of 3D NLSE where the nonlinearity is given by a negative cubic term (attractive two-body interactions). This can have experimental interest, as exemplified in Ref. [34], in which it has been investigated the dynamics of Bose-Einstein condensates submitted to anharmonic magnetic waveguides, with a polynomial trap.

Our aim, in the present work, is to make a threedimensional quantitative study on the limitations of $N_{c}$, for NLSE with attractive cubic term, with power-law trapping potentials of the form $\sim r^{\alpha}$. We assume the system in spherical symmetry. This work extends a previous one on anharmonic trap potentials [22], where we have obtained the maximum critical number of condensed atoms, for an original harmonic potential altered by the addition of cubic and quartic terms as perturbations. In the perspective of different other possibilities to obtain experimentally anharmonic traps for systems with attractive two-body interactions, we have analyzed power-law potentials that deviate from the harmonic one by a multiplicative factor. Besides the cubic nonlinear term in the NLSE, we have also considered a positive quintic term, due to elastic three-body interactions. In this case, as reported in Refs. [35,36], it is possible the occurrence in the system of a first order phase transition, that is a type of liquid gas phase transition. By type of liquid gas phase transition we mean a first order phase transition in $T=0$ where density changes abruptly when the number of atoms is incremented.

Actually, at the phase transition, we study the behavior of the critical value of the strength $g_{3 c}$ of the quintic term as a function of the power $\alpha$ of the confining potential. An approximate relation between $g_{3 c}$ and $\alpha$ is found in this limit.

In the next section, we present the basic formalism (Sec. II A) and corresponding results (Sec. II B), considering the 
nonlinearity of the NLSE given by a negative cubic term (attractive two-body interactions). In Sec. III, we have included a positive nonlinear quintic term in our formalism, in the perspective to study possible phase transitions in the system. Finally, in Sec. IV, we present our conclusions and final remarks.

\section{CUBIC NLSE WITH POWER-LAW TRAPPING POTENTIAL}

\section{A. Formalism}

The Gross-Pitaevskii equation describes a quantum manybody problem for a very dilute gas [2-4]. In spherical symmetry, the corresponding mean-field equation, for attractive two-body interactions, has the following form:

$$
i \hbar \frac{\partial \Psi}{\partial t}=\left(-\frac{\hbar^{2}}{2 m} \vec{\nabla}^{2}+V_{\text {trap }}(r)-\frac{4 \pi \hbar^{2}|a|}{m}|\Psi|^{2}\right) \Psi,
$$

where $\Psi \equiv \Psi(\vec{r}, t)$ is the wave function and $V_{\text {trap }}$ the trapping potential. $a$ is the two-body $s$-wave scattering length, and $m$ is the mass of the atomic species. In the above form, the wave function $\Psi(\vec{r}, t)$ is normalized to the number $N$ of particles,

$$
\int|\Psi(\vec{r}, t)|^{2} d^{3} \vec{r}=N .
$$

In the specific case of power-law potentials, in spherical symmetry, we can write the trapping potential as

$$
V_{\text {trap }}(r)=\frac{\hbar \omega}{4}\left(\frac{\sqrt{2} r}{l_{0}}\right)^{\alpha},
$$

where $l_{0} \equiv \sqrt{\hbar /(m \omega)}$ defines a unit of length. For $\alpha=2$ we have the usual 3D harmonic oscillator potential with $\omega$ being a geometrical average of the frequencies in the three spatial directions $\left(\omega^{3} \equiv \omega_{1} \omega_{2} \omega_{3}\right)$.

The stationary solutions of Eq. (1) are given by the chemical potential $\mu$, by setting $\Psi(\vec{r}, t)=e^{-\mathrm{i} \mu t / \hbar} \psi(\vec{r})$, such that

$$
\left(-\frac{\hbar^{2}}{2 m} \vec{\nabla}^{2}+V_{\text {trap }}(r)-\frac{4 \pi \hbar^{2}|a|}{m}|\psi(\vec{r})|^{2}\right) \psi(\vec{r})=\mu \psi(\vec{r}) .
$$

The trap is spherically symmetric and we are interested in the ground-state solutions. So, in Eq. (4), we assume the system is in the $s$ wave, and redefine the wave function, the variable $r$ and the chemical potential $\mu$ as

$$
\Phi(x) \equiv \sqrt{8 \pi|a|} r \psi(\vec{r}), \quad x \equiv \frac{\sqrt{2}}{l_{0}} r, \quad \beta=\frac{\mu}{\hbar \omega} .
$$

Using the above, we have

$$
\beta \Phi(x)=-\frac{d^{2} \Phi(x)}{d x^{2}}+\frac{x^{\alpha}}{4} \Phi(x)-\frac{|\Phi(x)|^{2}}{x^{2}} \Phi(x) .
$$

As $\psi(\vec{r})$ is normalized to $N$, from (5) we obtain the corresponding normalization of $\Phi(x)$ as

$$
\int_{0}^{\infty} d x|\Phi(x)|^{2}=2 \sqrt{2} \frac{N|a|}{l_{0}} .
$$

The boundary conditions of (6) are such that $\Phi(0)=0$ and $\Phi(x \rightarrow \infty)=0$. The total energy of the system is given by

$$
\begin{gathered}
E_{\text {tot }}=\hbar \omega\left(\frac{l_{0}}{2 \sqrt{2}|a|}\right) \mathcal{E}, \\
\mathcal{E} \equiv \int_{0}^{\infty} d x\left\{\left|\frac{d \Phi(x)}{d x}\right|^{2}+\frac{x^{\alpha}}{4}|\Phi(x)|^{2}-\frac{|\Phi(x)|^{4}}{2 x^{2}}\right\} .
\end{gathered}
$$

The size can be obtained from the corresponding meansquare radius $\left\langle r^{2}\right\rangle$, that can be written in terms of a dimensionless observable $\left\langle x^{2}\right\rangle$, as

$$
\left\langle r^{2}\right\rangle=\frac{l_{0}^{2}}{2} \frac{\int_{0}^{\infty} d x x^{2}|\Phi(x)|^{2}}{\int_{0}^{\infty} d x|\Phi(x)|^{2}} \equiv \frac{l_{0}^{2}}{2}\left\langle x^{2}\right\rangle .
$$

By considering all possible variations of the power $\alpha$, in the above equations, one can analyze the behavior of the observables of the system in comparison with results obtained for the case of harmonic potential [35]. Of particular interest is the magnitude of the maximum critical number of particles $N_{c}$ in the condensate, as we vary the power $\alpha$.

Next, we present the numerical results for this section.

\section{B. Numerical results}

We have considered a full numerical approach to obtain our results for the spherically symmetrical equations given in the preceding section. In the present case of stationary solutions, we have applied the fourth-order Runge-Kutta method, which is combined with the shooting method in order to satisfy the boundary conditions (the method is described in Ref. [37]).

The Runge-Kutta method is used to integrate in space the function $\Phi(x)$, from $\Phi(0)$ to $\Phi\left(x_{\infty}\right)$, where $x_{\infty}$ is far away from the point that mimics infinity. To do so, we depart from given initial condition $\Phi(0)$, by shooting $\Phi^{\prime}(0)$ (unknown), and we propagate the function in $x$ until we get $\Phi\left(x_{\infty}\right)=0$. So, $\Phi\left(x_{\infty}\right)$ is a function of $\Phi^{\prime}(0)$, and we have a simple algebraic equation $\Phi\left(x_{\infty}\right)=F\left[\Phi^{\prime}(0)\right]$. It is interesting to observe that if this function $F$ was known analytically, one could use the Newton-Raphson method, however in this case we only have this function numerically after propagating $\Phi$ from 0 to $\infty$, so that we had to use the secant method. These techniques are fully described in Ref. [37] and references therein [38].

The results of our calculations are presented for the total energy (Fig. 1), for the chemical potential (Fig. 2), and for the mean-square radius (Fig. 3). In Fig. 1 we have the results for the total energy $E_{\text {tot }}$. It is plotted $\mathcal{E}$, as given in Eq. (8), as a function of the number of particles $N$ scaled to a reduced number by a factor $|a| / l_{0}$. We show results from the case of linear confining potential $(\alpha=1)$ to the case of $\alpha=2.8$. As shown, the number of particles increases as we go to smaller values of the power $\alpha$. It is important to say that, although such a result is expected $\left(N_{c}\right.$ should increase for decreasing 


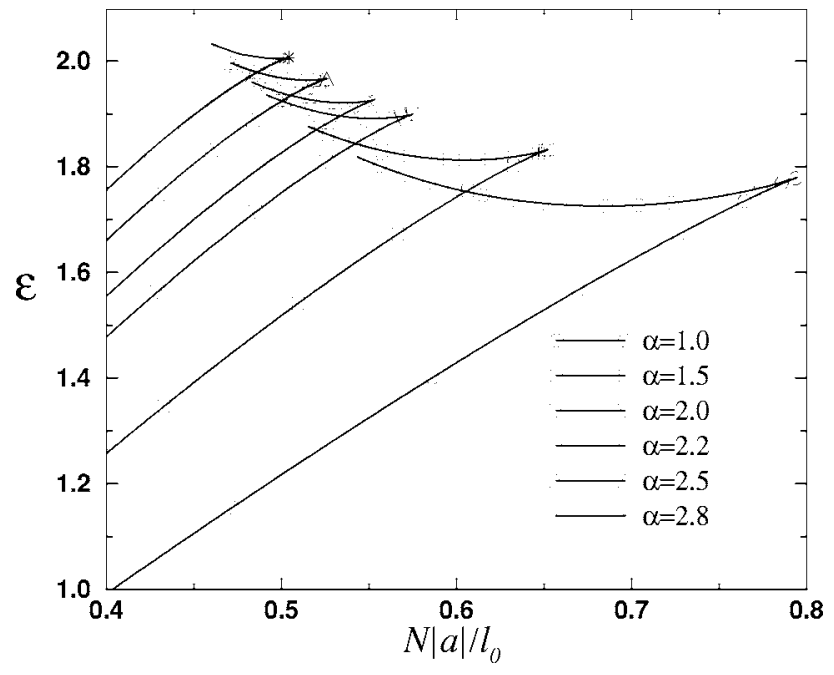

FIG. 1. The total energy $E_{\text {tot }}$, given by the corresponding dimensionless observable $\mathcal{E}$ [see Eq. (8)], versus the number of particles $N$, scaled by a factor $|a| / l_{0}$. The numerical results are shown for different confining power-law potentials, Eq. (3), with $\alpha=1.0$ to $\alpha$ $=2.8$, as indicated inside the figure.

$\alpha$ ), we need to quantify the increasing in the critical number of particles. So, we proceed to this calculation and we verify that, unless other technical problems, a linear trap could condensate a significant higher number of atoms, up to $40 \%$ higher than the harmonic one, in the attractive cases.

The lower branches of the curves correspond to the stable solutions of Eq. (8). Such curves reach critical points in the extreme right (collapse points), from where the solutions become unstable. The upper branches of the curves correspond to the unstable solutions (see, for instance, Ref. [3], Fig. 12, for a variational analysis). Both, stable and unstable static

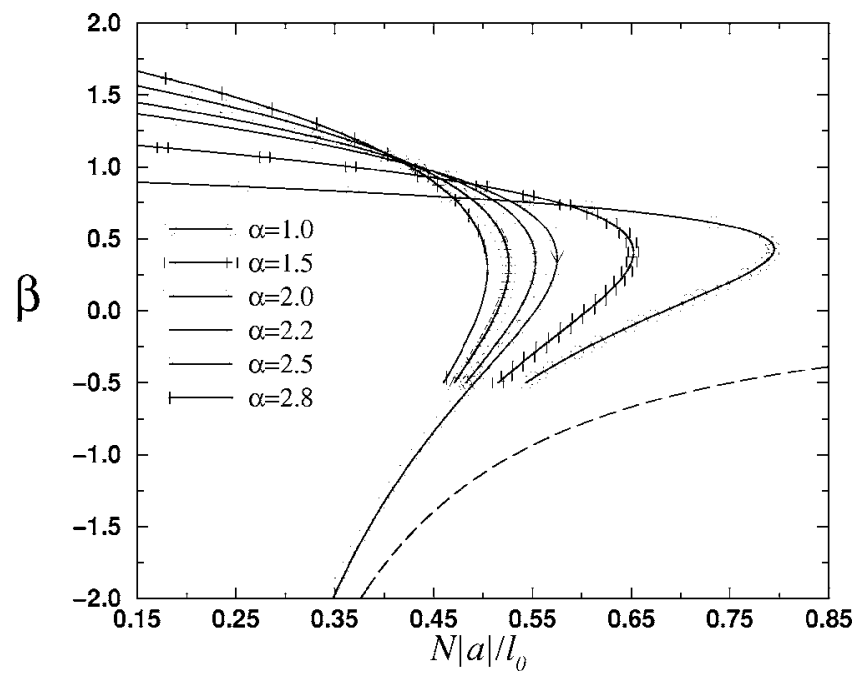

FIG. 2. The chemical potential $\mu$, given by the corresponding dimensionless observable $\beta[=\mu /(\hbar \omega)]$, versus the number of particles $N$, scaled by a factor $|a| / l_{0}$. The numerical results are shown for different confining power-law potentials, Eq. (3), with $\alpha=1.0$ to $\alpha=2.8$, as indicated inside the figure. For comparison, we have included the solutions of the free focusing NLSE, that is, the free focusing NLSE limit.

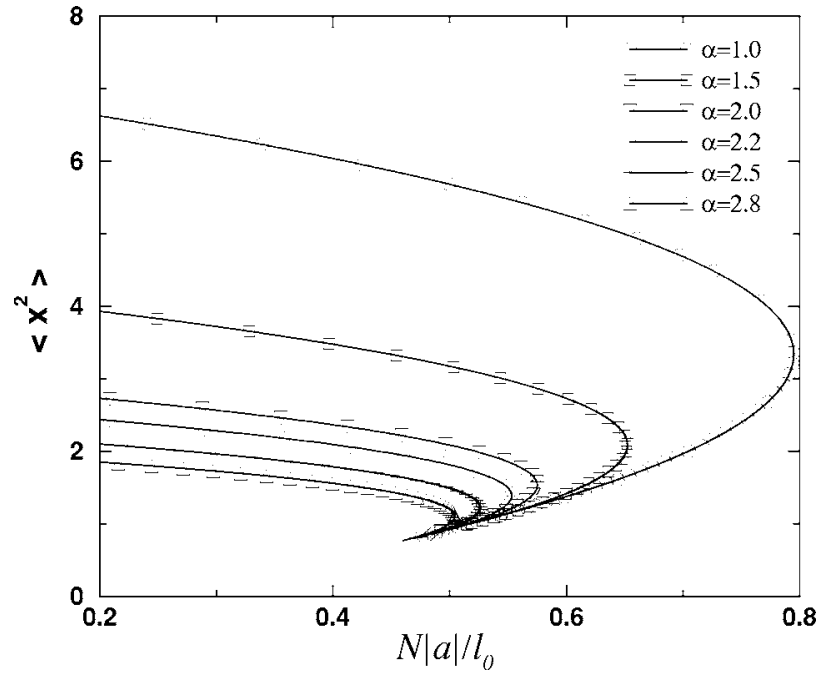

FIG. 3. The mean-square radius $\left\langle r^{2}\right\rangle$, given by the corresponding dimensionless observable $\left\langle x^{2}\right\rangle$, as defined in Eq. (9), versus the number of particles $N$, scaled by a factor $|a| / l_{0}$. The numerical results are shown for different confining power-law potentials, Eq. (3), with $\alpha=1.0$ to $\alpha=2.8$, as indicated inside the figure.

solutions are relevant to know in a stability analysis of the system. By varying $N$ for a given $\alpha$, the stable solution for the energy reaches its maximum, $\mathcal{E}_{c}$, at the critical point. The maximum number of condensed particles increases as the potential becomes less confining. For the case of linear trapping potential, we obtain $N_{c}|a| / l_{0} \cong 0.795$, corresponding to $\mathcal{E}_{c} \cong 1.78$. By comparing with the result obtained for the harmonic trapping potential $(\alpha=2)$, which is $N_{c}|a| / l_{0}=0.575$, we note that the linear case represents an increase in the maximum number of particles of about $38 \%$.

In Fig. 2, we present results for the chemical potential $\mu$, given by the corresponding dimensionless observable $\beta$, as a function of the number of atoms $N$, scaled by the factor $|a| / l_{0}$. As in Fig. 1, each of the curves are composed by a stable branch (upper part) and an unstable branch (lower part), that are joined at the critical points. The behavior of the curves is qualitatively similar for all the cases. However, if we fix the number of particles, we note that the values of $\beta$ are smaller on the stable parts of the curves for smaller values of the power $\alpha$ only when the scaled number of particles, $N|a| / l_{0}$, is not too large. It can happen an inversion of this behavior near the critical limits of potentials with higher powers. This happens because the critical numbers $N_{c}$ are smaller for larger values of $\alpha$. To extend the region of stability to higher number of particles, we must decrease significantly the power of the trapping potential. We also plot in Fig. 2 the curve of the focusing NLSE with no trap (free focusing NLSE), whose unstable solutions and singularity at finite time were verified for the first time about 30 years ago by Zakharov and Shabat [39]. In fact, in Fig. 2, we compared the variation of the scaled number of particles of the system versus the variation of the chemical potential for the free focusing NLSE. In these 3D systems, we note that the number of particles for all of the trapped cases always lies below the particle number of the free case and, in the limit $\beta \rightarrow \infty$, the trapped systems behaves as free NLS solitons [40]. In 


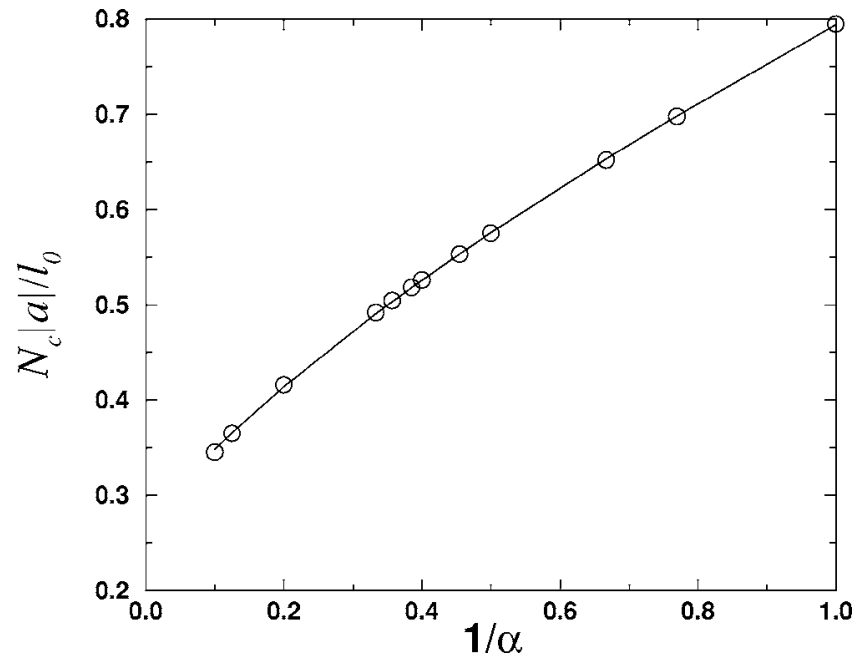

FIG. 4. The critical number of atoms $N_{c}$, scaled by the factor $|a| / l_{0}$, as a function of the inverse power of the trapping potential. The solid line is interpolating the numerical results, represented by circles.

Fig. 3, we present our numerical results for the mean-square radius $\left\langle r^{2}\right\rangle$, given by the corresponding dimensionless observable $\left\langle x^{2}\right\rangle$. For a given atomic system with negative twobody scattering length, as we increase the number of particles $N$ the stable condensate becomes smaller, shrinking until its minimum size at the critical point (extreme righthand-side of each curve with fixed $\alpha$ ). From the critical points, the curves also show the respective sizes of the unstable solutions (lower branches), corresponding to maxima for the energies. By comparing results from the harmonic oscillator confining potential $(\alpha=2)$ with the linear one $(\alpha=1)$, we observe a considerable increase in the meansquare radius at the critical point: $\left\langle x^{2}\right\rangle_{\alpha=1} \cong 2\left\langle x^{2}\right\rangle_{\alpha=2}$. In Fig. 4 , we present our numerical results for the maximum critical number of particles for stable solutions, scaled by the factor $|a| / l_{0}$, as a function of the power $\alpha$ of the confining potential. With $\alpha$ decreasing, the scaled critical number of condensed atoms will increase. For the harmonic trap, in spherical symmetry, we reproduce the well-known critical number $N_{c}|a| / l_{0}=0.575$. In case of linear trap $(\alpha=1)$, we obtain $N_{c}|a| / l_{0} \cong 0.795$. Table I shows results for a few specific values of $\alpha$.

\section{NLSE WITH THREE-BODY ELASTIC INTERACTIONS}

In this section we report our results when considering cubic and quintic terms in the NLSE, with a power-law potential trap. In spherical symmetry, using dimensionless variables as in (5), we obtain an extended version of Eq. (6), with $\Phi \equiv \Phi(x)$, such that

$$
-\frac{d^{2} \Phi}{d x^{2}}+\frac{x^{\alpha}}{4} \Phi-\frac{|\Phi|^{2}}{x^{2}} \Phi+g_{3} \frac{|\Phi|^{4}}{x^{4}} \Phi=\beta \Phi .
$$

In the above equation, the three-body dimensionless elastic interaction with strength $g_{3}$ is derived from the corresponding dimensional expression [36], as

$$
g_{3} \frac{|\Phi(x)|^{4}}{x^{4}} \hbar \omega=\lambda_{3}|\psi(\vec{r})|^{4},
$$

where $\lambda_{3}$ is the original strength of the quintic term. From Eqs. (5) and (11), we have the relation between $g_{3}$ and $\lambda_{3}$,

$$
g_{3}=\frac{\lambda_{3}}{\hbar \omega}\left(\frac{1}{4 \pi|a| l_{0}^{2}}\right)^{2} \text {. }
$$

In Eq. (10), we assume that $g_{3}$ is positive; and, as in the preceding section, the cubic term corresponds to attractive two-body interactions.

As we will see next, the system can exhibit a first order phase transition, called liquid-gas phase transition, that occurs in this model when we vary the number of particles and, therefore, the density of the system. Here, we are concerned specifically with the influence of deviations from the harmonic trap in the observed phase transition that was previously studied in Refs. [35,36]. For the case of attractive twobody interactions, it was reported that a positive nonlinear quintic term $\left(g_{3} \neq 0\right)$ allows the number of condensed particles to be larger than the $N_{c}$ obtained when $g_{3}=0$. It is further observed that a liquid-gas phase transition can occur in the system if $g_{3}$ is smaller than some critical value $g_{3 c}$. As shown in Ref. [36], for the harmonic trap potential, $g_{3 c}=0.0183$. Our aim is to verify the behavior of this critical value as we vary the shape of the trapping potential, using different powers $\alpha$ in Eq. (3).

In Fig. 5, we present our numerical results at the critical interface where the liquid-gas phase transition disappears. We present the results obtained for the strength of the quintic term $g_{3 c}$ and also for the corresponding scaled number of particles, $N|a| / l_{0}$, as functions of the inverse of the power $\alpha$ of the trapping potential. We should clarify that $N|a| / l_{0}$, in the small figure inserted in Fig. 5, corresponds to the value that we have when $g_{3}=g_{3 c}$. In Table II we also present the numerical results for some specific powers $\alpha$. There is no liquid-gas phase transition when $g_{3}>g_{3 c}$. The results show that, as we decrease the value of $\alpha$ we extend the region of parameters $g_{3}$ where it is possible to obtain liquid-gas phase transitions.

So, in the perspective that a condensed atomic system is such that we have a positive quintic term (nonlinear elastic three-body term) in the NLSE, with a less confining powerlaw potential we increase the upper limit of $g_{3}$ to obtain phase transitions. We observe that, in the case of linear trap-

TABLE I. Numerical results for the maximum critical value of the scaled number of particles, for a few values of $\alpha$ of the trapping potential.

\begin{tabular}{lcccccc}
\hline \hline$\alpha$ & 1.0 & 1.5 & 2.0 & 2.5 & 3.0 & 5.0 \\
\hline$N_{c}|a| / l_{0}$ & 0.795 & 0.652 & 0.575 & 0.526 & 0.492 & 0.416 \\
\hline \hline
\end{tabular}




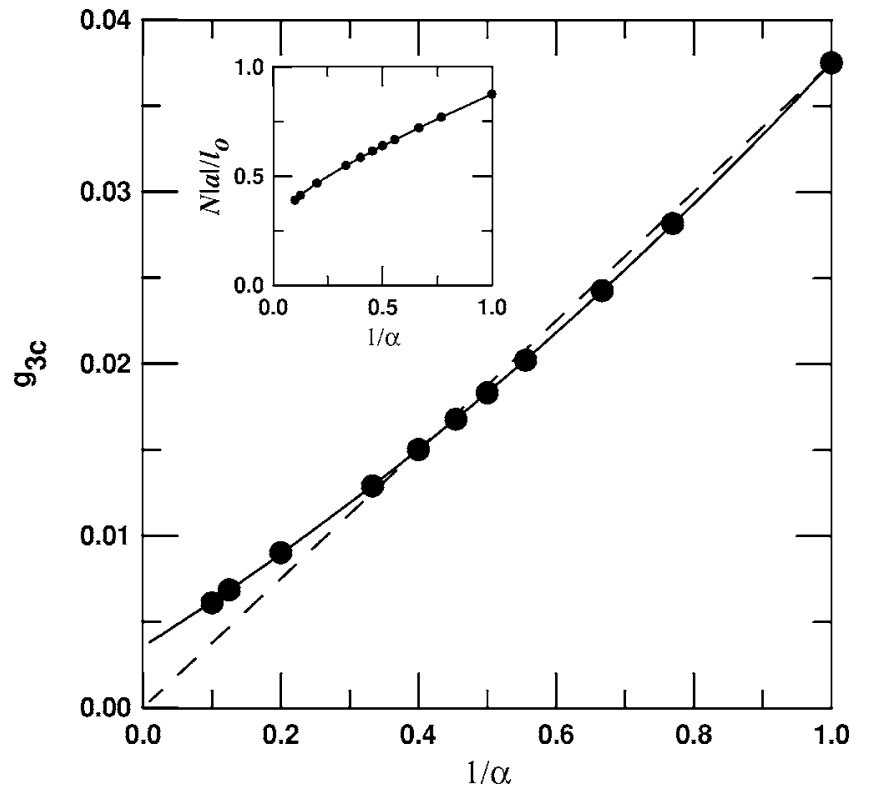

FIG. 5. Upper limit for the dimensionless three-body elastic parameter $g_{3}$ for the occurrence of liquid-gas phase transition as a function of the inverse of the trapping power $\alpha$. Our numerical results are represented by solid circles. The dashed line is a linear fit to such results $\left(g_{3 c} \cong 0.037 / \alpha\right)$. The solid line corresponds to the fitted expression, given by Eq. (13). The inset shows the corresponding scaled number $N|a| / l_{0}$ as a function of $\alpha$.

ping potential $(\alpha=1)$ we have $g_{3 c} \cong 0.037$, which is about 2 times the value obtained for the case of harmonic potential [36] $\left(g_{3 c}=0.0183\right)$. Observing that $g_{3 c}=0.0129$ when $\alpha=3$, a simple expression like $g_{3 c} \cong 0.037 / \alpha$ can almost fit the region $1 \leq \alpha \leq 3$ (see Fig. 5). A better fit for the behavior of $g_{3 c}$ with respect to $1 / \alpha$, at the phase transition, is given by

$$
g_{3 c} \sim 0.0036+\frac{0.0251}{\alpha}+\frac{0.0088}{\alpha^{2}} .
$$

When $g_{3}<g_{3 c}$, the scaled number of particles is a function of the chemical potential $\beta$ with two local extrema: one maximum and one minimum. At the critical point, where $g_{3}=g_{3 c}$, these two extrema disappear (see Ref. [35]). As the first and second derivative of $N|a| / l_{0}$ in terms of $\beta$ are zero, a relation between $\alpha$ and $g_{3 c}$ can be established. The above relation is an approximation that relies on a precise fitting of our numerical results, considering $1 \leq \alpha \leq 10$.

We note that, when $1 / \alpha \rightarrow 0$, the trap potential tends to a spherical potential well. The trapping potential is almost zero inside the well to be compared with the nonlinear terms; and becomes infinite at some finite radius, where the wave function goes to zero. From Eq. (13)), the limit $\alpha \rightarrow \infty$ implies $g_{3 c} \sim 0.0036$. In the other limit, when $\alpha=0$, there is no confining potential, as it becomes just a constant in the Hamiltonian. The effective potential is nonlinear, combining a negative cubic with a positive quintic term. In this case, the strength $g_{3}$ is not limited.

The liquid-gas phase transition, in principle, could be observed in experiments with certain atomic species with repulsive three-body interactions, near a Feshbach resonance where one can vary the two-body scattering length from positive to negative values. As explained in Refs. [35,36], the characterization of two phases can be done through the energies, chemical potentials, central densities or radius. As we increase the number of atoms in a experiment, instead of a collapsing behavior one should verify the system shrinking to a smaller radius. It is also shown in Ref. [36] that the decay time of the condensate that begins in a denser phase can be long enough to allow observation.

\section{CONCLUSIONS}

In summary, we have solved a stationary NLSE with cubic and quintic nonlinear terms and with an anharmonic trapping power-law potential. We have verified and quantified the increasing in the critical number of particles by changing the geometry of the trap, and the critical limit in which occurs a first order phase transition internal to the condensate due to elastic collisions for each power $\alpha$ of the power-law confining potential. In the first part of our work, the calculations are given in spherical symmetry and the nonlinearity was given by just the cubic term, corresponding to an attractive two-body interaction. As in this case there is no stable solutions when the scaled number of particles $N|a| / l_{0}$ is larger than some critical value, we study this critical limit $N_{c}|a| / l_{0}$ as a function of the power $\alpha$ of the confining potential. We present results for the total energy, the chemical potential, and the mean-square radius. We conclude that the maximum number of condensed particles increases as the potential becomes less confining. For the case of linear trapping potential, $N_{c}|a| / l_{0} \cong 0.795$, which is about $38 \%$ larger than the harmonic one $(0.575)$.

A repulsive elastic three-body interaction (positive quintic term) is added in our analysis, in Sec. III, to verify the possible occurrence of liquid-gas phase transitions. We extended a previous analysis of the phase transitions, that was done in Refs. [35,36] for harmonic trapping potential. When we have nonlinearity given by a negative cubic term, by decreasing the power $\alpha$ (to a less confining potential) we also allow the

TABLE II. Numerical results for a few values of $\alpha$ of the trapping potential. $g_{3 c}$ is the value of $g_{3}$ at the critical limit where the liquid-gas phase transition disappears. Equation (12) relates $g_{3}$ with the original strength $\lambda_{3}$ of the quintic term. $N$ is the corresponding number of particles.

\begin{tabular}{lllllll}
\hline \hline$\alpha$ & 1.0 & 1.5 & 2.0 & 2.5 & 3.0 & 5.0 \\
\hline$g_{3 c}$ & 0.0375 & 0.0242 & 0.0183 & 0.0150 & 0.0129 & 0.0090 \\
$N|a| / l_{0}$ & 0.876 & 0.722 & 0.639 & 0.586 & 0.549 & 0.468 \\
\hline \hline
\end{tabular}


number of particles to increase. By including a positive quintic term, there is no upper limit for the number of condensed particles, but some critical region where a liquid-gas phase transition can occur. Such a phase transition can occur only when the quintic term is not a dominant term in the effective interaction, such that the corresponding strength $g_{3}$ is limited. In order to have liquid-gas phase transition we must have $g_{3} \leq g_{3 c}$, which is plotted as a function of $1 / \alpha$ in Fig. 5 . So, $g_{3 c}$ gives us the limiting value of $g_{3}$ where the three extremes of the energies (two minima and one maximum) disappear. We have determined numerically the values of $g_{3 c}$ for several values of $\alpha$ and, finally, found a quadratic analytical relation between $g_{3 c}$ and $1 / \alpha$, as shown in Eq. (13).
This equation is expected to be a good approximation to an expression between $g_{3 c}$ and $\alpha$, when we consider phase transitions in NLSE with confining power-law potential and with the nonlinearity given by negative cubic and positive quintic terms.

\section{ACKNOWLEDGMENTS}

This work was partially supported by Fundação de Amparo à Pesquisa do Estado de São Paulo (FAPESP) and Conselho Nacional de Desenvolvimento Científico e Tecnológico (CNPq) do Brazil.
[1] I. S. Aranson and L. Kramer, Rev. Mod. Phys. 74, 99 (2002).

[2] P. W. Courteille, V. S. Bagnato, and V. I. Yukalov, Laser Phys. 2, 659 (2001).

[3] F. Dalfovo, S. Giorgini, L. P. Pitaevskii, and S. Stringari, Rev. Mod. Phys. 71, 463 (2001).

[4] A. S. Parkins and D. F. Walls, Phys. Rep. 303, 1 (1998).

[5] V. L. Ginzburg and L. P. Pitaevskii, Zh. Eksp. Teor. Fiz. 34, 1240 (1958) [Sov. Phys. JETP 7, 858 (1958)]; L. P. Pitaevskii, ibid. 40, 646 (1961) L. P. Pitaevskii, [ ibid. 13, 451 (1961)]; E. P. Gross, J. Math. Phys. 4, 195 (1963).

[6] M. Edwards and K. Burnett, Phys. Rev. A 51, 1382 (1995); P. A. Ruprecht, M. J. Holland, K. Burnett, and M. Edwards, ibid. 51, 4704 (1995).

[7] E. Demircan, P. Ao, and Q. Niu, Phys. Rev. B 54, 10027 (1996); C. D. Levermore and D. R. Stark, Phys. Lett. A 234, 269 (1997); J. Koplik and H. Levine, Phys. Rev. Lett. 71, 1375 (1993); D. P. Arovas and J. A. Freire, Phys. Rev. B 55, 1068 (1997).

[8] M. H. Anderson, J. R. Ensher, M. R. Matthews, C. E. Wieman, and E. A. Cornell, Science 269, 198 (1995).

[9] C. C. Bradley, C. A. Sackett, J. J. Tollett, and R. G. Hulet, Phys. Rev. Lett. 75, 1687 (1995).

[10] F. Dalfovo, C. Minniti, S. Stringari, and L. P. Pitaevskii, Phys. Lett. A 227, 259 (1997).

[11] W. Ketterle, Physica B 280, 11 (2000).

[12] H.-J. Miesner and W. Ketterle, Solid State Commun. 107, 629 (1998).

[13] S. Yi and L. You, Phys. Rev. Lett. 92, 193201 (2004); Phys. Rev. A 67, 045601 (2003).

[14] C. Huepe, L. S. Tuckerman, S. Métens, and M. E. Brachet, Phys. Rev. A 68, 023609 (2003).

[15] L. P. Pitaevskii, Phys. Lett. A 229, 406 (1997); L. P. Pitaevskii, ibid. 221, 14 (1996).

[16] Y. Kagan, A. E. Muryshev, and G. V. Shlyapnikov, Phys. Rev. Lett. 81, 933 (1998).

[17] V. S. Filho, A. Gammal, T. Frederico, and L. Tomio, Phys. Rev. A 62, 033605 (2000); V. S. Filho, T. Frederico, A. Gammal, and L. Tomio, Phys. Rev. E 66, 036225 (2002).

[18] S. K. Adhikari, Phys. Lett. A 296, 145 (2002); S. K. Adhikari, Phys. Rev. A 66, 013611 (2002).

[19] E. A. Donley et al., Nature (London) 412, 295 (2001).

[20] A. Gammal, L. Tomio, and T. Frederico, Phys. Rev. A 66,
043619 (2002); A. Gammal, T. Frederico, and L. Tomio, ibid. 64, 055602 (2001).

[21] H. Michinel, V. Pérez-García, and R. de la Fuente, Phys. Rev. A 60, 1513 (1999); V. M. Pérez-García, H. Michinel, and H. Herrero, ibid. 57, 3837 (1998).

[22] V. S. Filho, A. Gammal, and L. Tomio, Phys. Rev. A 66, 043605 (2002).

[23] V. Bagnato, D. E. Pritchard, and D. Kleppner, Phys. Rev. A 35, 4354 (1987).

[24] P. W. H. Pinkse, A. Mosk, M. Weidemüller, M. W. Reynolds, T. W. Hijmans, and J. T. M. Walraven, Phys. Rev. Lett. 78, 990 (1997).

[25] S. Pearson, T. Pang, and C. Chen, Phys. Rev. A 58, 1485 (1998)

[26] M. Bayindir, B. Tanatar, and Z. Gedik, Phys. Rev. A 59, 1468 (1999).

[27] M. Li and Y. Z. Wang, Phys. Rev. A 64, 045601 (2001).

[28] Z. Yan, Phys. Rev. A 59, 4657 (1999).

[29] W.-S. Dai and M. Xie, Phys. Rev. A 67, 027601 (2003).

[30] L. Salasnich, J. Math. Phys. 41, 8016 (2000); L. Salasnich, Int. J. Mod. Phys. B 16, 2185 (2002).

[31] E. Lundh, Phys. Rev. A 65, 043604 (2002).

[32] H. Shi and W.-M. Zheng, Phys. Rev. A 56, 2984 (1997).

[33] O. Mülken, P. Borrmann, J. Harting, and H. Stamerjohanns, Phys. Rev. A 64, 013611 (2001).

[34] H. Ott, J. Fortágh, S. Kraft, A. Günther, D. Komma, and C. Zimmermann, Phys. Rev. Lett. 91, 040402 (2003).

[35] A. Gammal, T. Frederico, L. Tomio, and Ph. Chomaz, J. Phys. B 33, 4053 (2000).

[36] A. Gammal, T. Frederico, L. Tomio, and Ph. Chomaz, Phys. Rev. A 61, 051602(R) (2000).

[37] A. Gammal, T. Frederico, and L. Tomio, Phys. Rev. E 60, 2421 (1999).

[38] D. Quinney, An Introduction to the Numerical Solution of Differential Equations (Wiley, New York, 1987), p. 133.

[39] V. E. Zhakarov and A. B. Shabat, Zh. Eksp. Teor. Fiz. 61, 118 (1971) [Sov. Phys. JETP 34, 62 (1972)]; V. E. Zhakarov, ibid. 62, 1745 (1972) V. E. Zhakarov, [Sov. Phys. JETP 35, 908 (1972)].

[40] L. Bergé, T. J. Alexander, and Yu. S. Kivshar, Phys. Rev. A 62, 023607 (2000). 